\title{
Exaggerated Pigmented Granulomatous Reaction to the Artificial Joint Implant Mimics Metastatic Melanoma
}

Wei Xin, M.D., Ph.D., ${ }^{1}$ Robertson D. Davenport, M.D., ${ }^{1}$ Alfred E. Chang, M.D., ${ }^{2}$ and Claire W. Michael, M.D. ${ }^{1 *}$

Total joint replacement is a common orthopedic procedure. An artificial joint implant may fail due to mechanical mishap and a granulomatous reaction can be induced by the artificial joint debris after the mechanical failure. We report a case of an exaggerated pigmented granulomatous tissue response to metallic artificial joint implant debris in a 72-yr-old male that was mistaken for metastatic melanoma. The mass was soft, pigmented, ill-defined, and located in the right inguinal region. Fine-needle aspiration revealed numerous black-pigment laden cells. The cellular features were frequently obscured by the heavy pigmentation. Occasional cells exhibited atypia and prominent nucleoli. There were also abundant extracellular irregular small black particles dispersed in the background. The diagnosis of melanoma involving a lymph node was made. Since there was no prior history of melanoma, it was presumed that this represented metastatic melanoma from an unknown primary. A subsequent exploration of the groin was performed with the intent to resect the disease. At exploration, the mass was found to be contiguous with the hip joint and the frozen section of the mass revealed no evidence of melanoma. The final tissue diagnosis confirmed the frozen section report and showed a granulomatous reaction. This report underscores the diagnostic dilemma associated with the exaggerated pigmented granulomatous reaction due to an artificial prosthesis. Diagn. Cytopathol. 2004;30:198-200.

๑ 2004 Wiley-Liss, Inc.

Key Words: artificial joint replacement; pigmented tissue response; cytology

Malignant melanoma should always be considered in the differential diagnosis of a fine-needle aspirate (FNA) that

${ }^{1}$ Department of Pathology, The University of Michigan Medical Center, Ann Arbor, Michigan

${ }^{2}$ Department of Surgery, The University of Michigan Medical Center, Ann Arbor, Michigan

*Correspondence to: Claire W. Michael, MD, Department of Pathology, The University of Michigan Hospitals, 1500 E. Medical Center Dr., Ann Arbor, MI 48109. E-mail: clairemi@med.umich.edu

Received 3 April 2003; Accepted 9 September 2003

DOI $10.1002 / \mathrm{dc} .20006$

Published online in Wiley InterScience (www.interscience.wiley.com). contains heavily pigmented cells. However, the differential diagnosis of an aspirate from a soft tissue mass adjacent to a joint prosthesis should include other lesions, such as pigmented villonodular synovitis and detritic synovitis. ${ }^{1,2}$ This report illustrates a case of detritic synovitis mistaken for metastatic melanoma in a patient with a hip prosthesis.

\section{Case Report}

A 72-yr-old white male patient presented with an enlarged right inguinal mass for 4 mo. The mass was not painful but it had a black appearance. His past medical history was significant for bilateral total hip arthroplasty $10 \mathrm{yr}$ ago, coronary artery disease status postmyocardial infarction with a blockage of his left anterior descending artery, status post unsuccessful coronary artery angioplasty, and benign prostatic hypertrophy. Physical examination revealed a $3.0-\mathrm{cm}$ ill-defined mass located above the right inguinal crease. The mass was not movable and adherent to the deep and surrounding soft tissue. A CT scan of the chest, abdomen, and pelvis showed a pelvic mass in the right iliopsoas area of attenuated signal of unknown significance. The subsequent MRI of the pelvis revealed right inguinal lymphadenopathy suspicious for malignancy. An FNA was performed for diagnostic purposes. Microscopically, the aspirate consisted of numerous black-pigment-laden cells which were pleomorphic and contained prominent nucleoli. The majority of the cells were completely obscured by the intracellular black pigment. There were also abundant extracellular black small particles dispersed in the background. Normal-appearing small lymphocytes were also present in the background (Fig. 1). The diagnosis of malignant melanoma was made. Since the patient had not had a previous history of a primary melanoma, it was presumed that this represented nodal metastases from an unknown primary melanoma site. A thorough examination for a pri- 

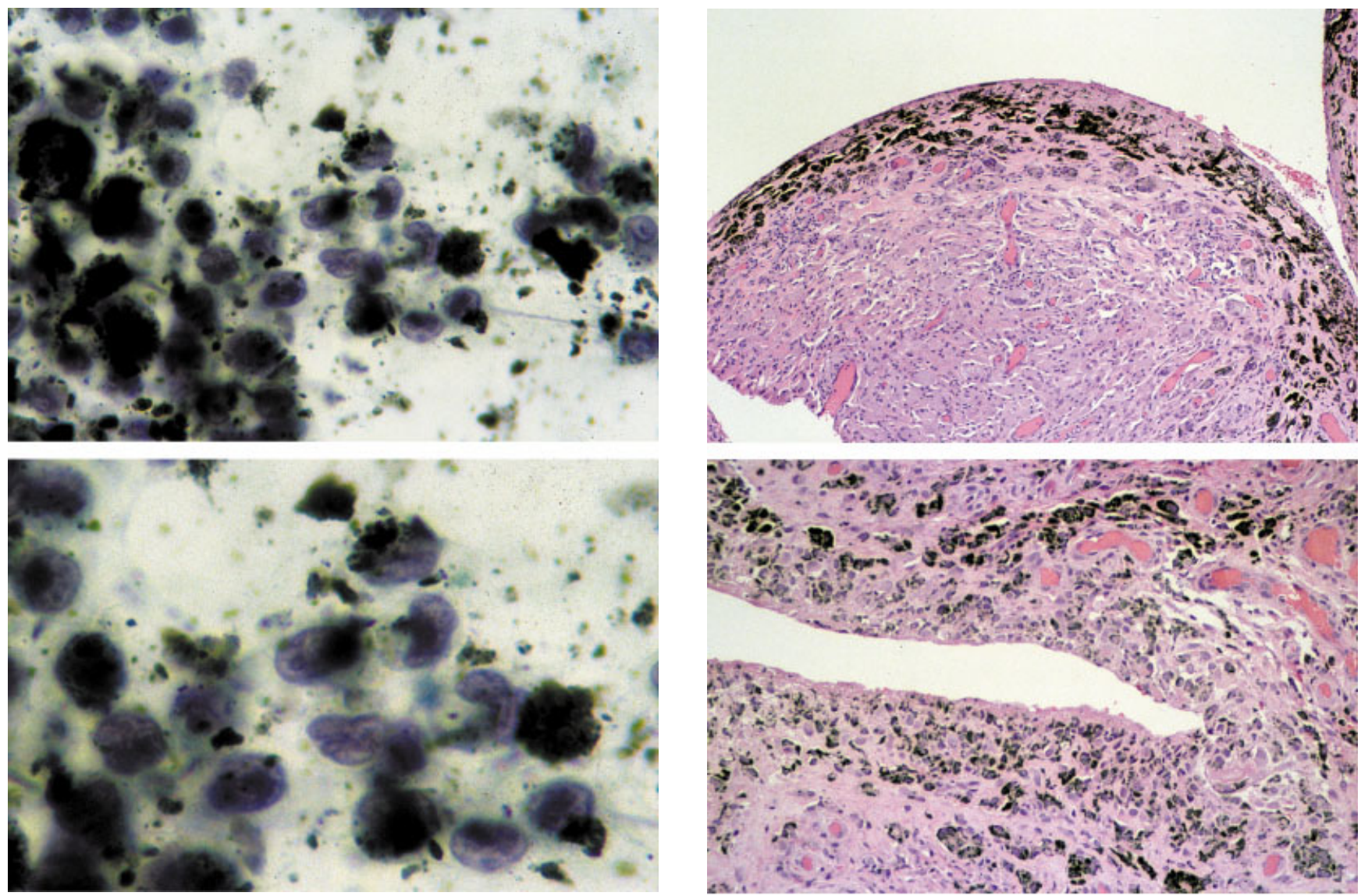

Fig. 1. Black-pigment-laden large atypical cells obtained by FNA. Papanicolaou stain. A: Low magnification. B: High magnification.

mary was negative. A superficial and deep inguinal lymph node dissection was recommended for curative therapy.

At operation, what was presumed to be bulky, enlarged lymph nodes adjacent to the femoral nerve and vein began to extrude black fluid and fragments of polyethylene. The frozen section of a portion of the tissue revealed synovium with black-pigment-laden macrophages, granulomatous inflammation, and no evidence of melanoma (Fig. 2). The dissected tissue was removed and the operation terminated. Grossly, the resected specimen was an undefined, black fatty-looking soft tissue and attached portion of black synovium with relative homogenous cut surfaces. No hemorrhage, necrosis, or definite mass was identified. Eleven nonpigmented lymph nodes ranging from $0.5-3.0 \mathrm{~cm}$ were examined and had no evidence of melanoma. The case was diagnosed as granulomatous inflammation due to metal debris. The patient recovered from the surgery and underwent replacement of both hip prostheses at a later date.

\section{Comment}

Large joint arthroplasty is a common elective orthopedic procedure. The artificial joint implant could fail either due
Fig. 2. Hematoxylin-eosin-stained tissue sections. A: $\times 100$ magnification. B: $\times 200$ magnification.

to infection or mechanical mishap. With time, the mechanical erosion of the prosthesis can release particles. A foreign body reaction can be induced by the artificial joint implant debris. ${ }^{1,2}$ There are three major debris constituents: metallic, high-density polyethylene, and methyl methacrylate components. Metallic debris presents as small $1-3 \mu \mathrm{m}$ grayblack pigments, commonly seen within histiocytes. Highdensity polyethylene particles are pale and thread-like or needle-like birefringent shapes up to 10 or $20 \mu \mathrm{m}$ within histiocytes and giant cells. Methyl methacrylate is not seen directly in histologic sections due to extraction during processing. Detritic synovitis is a term to describe the soft tissue response to artificial joint implants. It typically manifests as red-tan nodular synovial thickening. Black pigmentation due to metal debris may be seen. The intense histiocytic and foreign body giant cell reaction is seen in tissue sections..$^{3-6}$ Metallic debris often is not conspicuous and may be easily overlooked. Metallic particles usually are less than $1 \mu \mathrm{m}$ and ingested by histiocytes. They present as refractile black particles in the histiocytic cytoplasm.

When a cytopathologist screens an FNA with heavily pigmented cells from a mass in proximity to a joint implant, 
XIN ET AL.

Table I. Characteristics of Pigments

\begin{tabular}{llcl}
\hline Pigment & \multicolumn{1}{c}{ Papanicolaou stain } & Polarization & \multicolumn{1}{c}{ Special stain } \\
\hline Metalic debris & Black. Irregular in shape and variable in size. & + & None \\
Hemosiderin & Golden-brown. Refractile and fine granular. & Iron \\
Melanin & Dark brown. Finely granular and diffusely packed in & - & S100, HMB45, Melanin A. \\
& melanoma cells. Coarsely granular and densely & - & (Melanin bleach if necessary). \\
& packed in histiocytes. & & \\
\hline
\end{tabular}

she/he should not confuse this pigmented reaction with malignant melanoma. Other lesions that should be considered in the differential diagnosis include pigmented villonodular synovitis (PVNS) and hemosiderotic synovitis. PVNS is a locally aggressive tumor of synovium and tendon sheaths which can be either multiple or solitary. The lesion may be either painful or asymptomatic. Characteristically, PVNS has a nodular growth pattern and consists of collagen-producing polyhedral epithelioid cells with scattered giant cells and abundant hemosiderin-laden synovial lining cells. The hemosiderin is a golden-brown refractile pigment seen by Papanicolaou stain and can be confirmed by iron stain. ${ }^{1,7,8}$ Hemosiderotic synovitis occurs mainly in patients with chronic intraarticular bleeding and contains a large amount of hemosiderin pigments. Cytologically, these lesions would be expected to yield numerous hemosiderinladen macrophages. Similar to PVNS, the hemosiderin can be confirmed by iron stain. Melanotic melanomas may contain abundant intracellular black melanin. The diagnosis of melanoma should be based on the characteristic cytological features: mainly plasmocytoid pleomorphic loosely associated cells with macronucleoli, intranuclear inclusions, and nuclear membrane irregularity. Typically, melanin pigment is finely granular and diffusely distributed throughout cytoplasm of melanoma cells, but could be coarsely granular and densely packed in histiocytes. ${ }^{9}$ It is dark-brownish to black and can be confirmed by melanin A and/or HMB45 immunostains. In heavily pigmented lesions, bleaching may be necessary to reveal cytologic details.

In this case the FNA specimen contained abundant intracellular and extracellular pigmented particles with pleomorphic atypical cells. The differentiation among metal debris, hemosiderin, and melanin is summarized in Table I. In retrospect, the abundant extracellular pigmentation observed in the FNA specimen is not a classic presentation of melanoma. A careful examination of these particles would reveal that they are irregular and geometric in shape, variable in size, and refractile. Reactive histiocytes can exhibit cytologic atypia and should not be mistaken for malignancy. Despite the prominent nucleoli and bizarre shapes these histiocytes may present, they usually have bland nuclei with fine chromatin. Ordinary immunohistochemical stain for melanin A probably would not help in this case, since the stain reaction would not be recognizable against the heavily obscuring intracellular pigment. However, considering the long-standing history of hip arthroplasty and the polarizable pigment, a diagnosis of detritic synovitis reacting to metal debris should have been entertained. If the reactive process had been in the initial diagnosis workup, the melanin A bleach study as well as histiocytes markers should have been applied. A misdiagnosis of melanoma and the subsequent aggressive procedure might have been avoided. Nevertheless, if the distinction between melanoma and reactive changes is too difficult to make in an aspirate, an open biopsy to obtain more tissue should have been recommended to yield a correct diagnosis.

On the other hand, $\sim 15 \%$ of patients with melanoma present with metastatic disease to lymph nodes without a known primary site. Lymphadenectomy in these patients results in a similar survival to patients who undergo lymphadenectomy with known melanoma primary sites. ${ }^{10}$ Therefore, making a diagnosis of melanoma without a known primary is not uncommon, but should be cautious. In summary, this case illustrates that the artificial joint metal debris could induce a mass-like lesion and can be confused as malignant melanoma. A positive diagnosis should be based on definite cytologic features and should be avoided if the cells are obscured by the pigment.

\section{References}

1. Bullough PG. Joint diseases. In: Sternberg SS, et al., editors. Diagnostic surgical pathology, 3rd ed. Philadelphia: Lippincott Williams \& Wilkins; 1999. p 234-242.

2. O'Connell JX. Pathology of the synovium. Am J Clin Pathol 2000; 114:773-784.

3. Shokeir MO, Duncan CP, O'Connell JX. Revision hip arthroplasty: a histologic review. Int J Surg Pathol 1996;3:247-256.

4. Goldring SR, Clark CR, Wright TM. The problem in total joint arthroplasty: aseptic loosening. J Bone Joint Surg Am 1993;75:799801.

5. Mirra JM, Marder RA, Amstutz HC. The pathology of failed large joint arthroplasty. Clin Orthop 1990;261:140-152.

6. Zaloudek C, Treseler PA, Powell CB. Postarthroplasty histiocytic lymphoadenopathy in gynecologic oncology patients: a benign reactive process that clinically may be mistaken for cancer. Cancer 1996; 78:834-844.

7. Docken WP. Pigmented villonodular synovitis. Semin Arthritis Rheum 1979;9:1.

8. DeMay RM. The arts \& science of cytopathology: exfoliative cytology. Chicago: ASCP Press; 1996.

9. Masubuchi S Jr, Nagai I, Hirata M, Kubo H, Masubuchi K. Cytologic studies of malignant melanoma of the vagina. Acta Cytol 1975;19: 527-532.

10. Chang AE, Johnson TM, Rees RS. Cutaneous neoplasm. In: Greenfield LJ, et al., editors. Surgery-scientific principles and practice, 2 nd ed. Philadelphia: Lippincott-Ravens; 1997. p 2237-2258. 